\title{
REVIEW
}

\section{The role of peripheral nerve fibers and their neurotransmitters in cartilage and bone physiology and pathophysiology}

\author{
Susanne Grässel
}

\begin{abstract}
The peripheral nervous system is critically involved in bone metabolism, osteogenesis, and bone remodeling. Nerve fibers of sympathetic and sensory origin innervate synovial tissue and subchondral bone of diathrodial joints. They modulate vascularization and matrix differentiation during endochondral ossification in embryonic limb development, indicating a distinct role in skeletal growth and limb regeneration processes. In pathophysiological situations, the innervation pattern of sympathetic and sensory nerve fibers is altered in adult joint tissues and bone. Various resident cell types of the musculoskeletal system express receptors for sensory and sympathetic neurotransmitters. Osteoblasts, osteoclasts, mesenchymal stem cells, synovial fibroblasts, and different types of chondrocytes produce distinct subtypes of adrenoceptors, receptors for vasointestinal peptide, for substance $P$ and calcitonin gene-related peptide. Many of these cells even synthesize neuropeptides such as substance $P$ and calcitonin gene-related peptide and are positive for tyrosine-hydroxylase, the rate-limiting enzyme for biosynthesis of catecholamines. Sensory and sympathetic neurotransmitters modulate osteo-chondrogenic differentiation of mesenchymal progenitor cells during endochondral ossification in limb development. In adults, sensory and sympathetic neurotransmitters are critical for bone regeneration after fracture and are involved in the pathology of inflammatory diseases as rheumatoid arthritis which manifests mainly in joints. Possibly, they might also play a role in pathogenesis of degenerative joint disorders, such as osteoarthritis. All together, accumulating data imply that sensory and sympathetic neurotransmitters have crucial trophic effects which are critical for proper limb formation during embryonic skeletal growth. In adults, they modulate bone regeneration, bone remodeling, and articular cartilage homeostasis in addition to their classic neurological actions.
\end{abstract}

\section{Introduction}

Clinical observations demonstrated the importance of the peripheral nervous system for maintaining body homeostasis and mediating organogenesis and tissue repair. It is reported that in patients with head injuries, fractures frequently heal with excessive callus formation and at a faster rate than normal [1]. In patients with spinal cord injuries, a profound decrease of sublesional bone mineral density was measured in comparison with controls [2]. This loss of bone mass is higher at the distal femur and proximal tibia, which are the most common sites of fractures. In addition, hip fracture after stroke is an increasingly recognized problem. Measures to prevent bone loss, which

Correspondence: susanne.graessel@klinik.uni-regensburg.de

Department of Orthopaedic Surgery, Experimental Orthopaedics, University of Regensburg, ZMB/BioPark 1, Josef-Engert-Str. 9, 93053 Regensburg, Germany architecture have not been part of stroke management thus far [3].

These and other recent publications suggest that the peripheral nervous system is critically involved in bone metabolism, osteogenic differentiation of precursor cells (osteogenesis), bone mineralization, and bone remodeling $[4,5]$. Nerve fibers of sympathetic and sensory origin frequently innervate trabecular bone, periosteum, and fracture callus [6,7]. They are involved in controlling vascularization and matrix differentiation during endochondral ossification in embryonic limb development [8], indicating a distinct role in modulating skeletal growth and limb formation processes.

On one hand, disorders of nerves (central or peripheral) can have substantial influence on bone health, repair, and 
regeneration. On the other hand, dramatic alterations in density and distribution of sympathetic and sensory nerve fibers are reported in musculoskeletal pathophysiology. Changes in the density of sympathetic nerve fibers, which are characterized by tyrosine-hydroxylase $(\mathrm{TH})$ or neuropeptide Y (NPY) or both, in synovial tissue contribute to rheumatoid arthritis (RA) [9]. Loss of sensory joint innervation during aging is suggested to accelerate degenerative cartilage alterations which contribute to development of spontaneous osteoarthritis (OA) in mice [10]. Capsaicinsensitive sensory neurons contribute to the maintenance of tibial and femoral metaphyseal trabecular bone integrity and bone mass [11], suggesting a positive role in bone regeneration, whereas with respect to the sympathetic nervous system, some studies reported a favorable influence of $\beta$-blockers on bone mass and reducing fracture risk [12].

Sensory nerves in general contain two different nociceptive neuropeptide families: the tachykinins [13] and calcitonin gene-related peptides (CGRPs). Effects of all tachykinins are mediated by three neurokinin receptors with varying affinities for the individual ligands [14] (Table 1). Classically, tachykinin substance P (SP) is known as a mediator of nociception and of inflammation [15]. CGRP is encoded together with calcitonin and is generated by alternative splicing [16] and signals through a complex family of receptor proteins [17] (Table 1). The most important neurotransmitter of the catecholaminergic sympathetic nervous system is norepinephrine (NE), which signals through $\alpha$ - and $\beta$-adrenoceptors (ARs), depending on concentration [18] (Table 2). Vasoactive intestinal peptide (VIP) belongs to a family of structurally related peptides, including secretin, glucagon, gastric inhibitory peptide, growth hormone-releasing factor, and pituitary adenylate cyclase-activating polypeptide (reviewed in [19]). The three different subtypes of VIP receptors belong to the type II family of G protein-coupled receptors
(Table 2). Here, a concise overview of efferent functions and roles of sympathetic and sensory nerve fibers and their neurotransmitters in bone and cartilage physiology and pathophysiology is presented.

\section{Sensory and sympathetic nerve fibers in cartilage physiology and development}

In diarthrodial joints, permanent hyaline cartilage covers the surface of bones and enables them to bear very large compressive loads without distortion and allows smooth, frictionless movement of the joints [20]. Importantly, unlike other musculoskeletal connective tissues such as periosteum and synovium, cartilage does not contain blood vessels and is not deeply innervated by nerve fibers, indicating that cartilage for some reason might be a hostile environment for the spreading of nerve fibers. However, there is sparse evidence that sensory nerve fibers come into contact with those chondrocytes located in growth plate cartilage and in the outer layer of articular cartilage. In rat knee joints, CGRP-positive fibers which originate from periosteum and near insertion regions of muscle and tendons penetrate the outer layer of articular and meniscus cartilage up to $25 \mu \mathrm{m}$ and are located between single chondrocytes, indicating a local effector function. However, there are subpopulations of SP-positive axons in perichondrium and periosteum which for unknown reasons do not innervate the cartilage [21]. CGRP- and SP-positive nerve fibers precede the development of cartilage canals which are formed during skeletal growth shortly after birth and were detected when they penetrated the canals of growth cartilage in the epiphysis of young rats, thereby coming into close contact with chondrocytes [22,23]. The development of the secondary ossification center is subsequent to the presence of cartilage canals carrying sensory nerve fibers, and it is speculated that these nerve fibers modulate the formation of synovial joints through trophic effects

Table 1 Mammalian sensory neurotransmitters and their receptors

\begin{tabular}{|c|c|c|c|}
\hline Neurotransmitters & Receptor (highest affinity) & Neuropeptide genes & $\begin{array}{l}\text { Neuropeptide } \\
\text { receptor genes }\end{array}$ \\
\hline Substance P (SP) & Neurokinin $(\mathrm{NK})_{1}$ receptor & $\begin{array}{l}\text { Tachykinin (TAC) } 1 \text { or PPT-A } \\
\text { or PPT-I }\end{array}$ & Tachykinin receptor $(T A C R) 1$ \\
\hline Neurokinin A (NKA) & $\mathrm{NK}_{2}$ receptor & TAC1 & TACR2 \\
\hline \multicolumn{4}{|l|}{$\begin{array}{l}\text { Alternatively spliced forms are } \\
\text { neuropeptides (NP) K and NPY. }\end{array}$} \\
\hline Neurokinin B (NKB) & $\mathrm{NK}_{3}$ receptor & TAC3 or PPT-B or PPT-II & TACR3 \\
\hline Hemokinin 1 (HK1) & $\mathrm{NK}_{1}$ receptor, HK1 receptor? & TAC4 or PPT-C & TACR1 \\
\hline \multicolumn{4}{|l|}{$\begin{array}{l}\text { Alternatively spliced forms are } \\
\text { endokinins (EK) A, B, C, D. }\end{array}$} \\
\hline $\begin{array}{l}\text { Alpha-calcitonin-gene-related } \\
\text { peptide (aCGRP) }\end{array}$ & $\begin{array}{l}\text { Calcitonin receptor-like receptor (CRLR)/ } \\
\text { receptor activity-modifying protein (RAMP-1) }\end{array}$ & CALCA & CALCR/RAMP1 \\
\hline $\begin{array}{l}\text { Beta-calcitonin gene-related } \\
\text { peptide ( } \beta C G R P)\end{array}$ & CRLR/RAMP-1 & $C A L C B$ & CALCR/RAMP1 \\
\hline
\end{tabular}


Table 2 Mammalian sympathetic neurotransmitters and their receptors

\begin{tabular}{|c|c|c|c|}
\hline Neurotransmitters & Receptors & Neuropeptide genes & Neurotransmitter receptor genes \\
\hline \multicolumn{4}{|l|}{ Catecholaminergic } \\
\hline \multirow[t]{3}{*}{ Norepinephrine or noradrenaline (NA) } & $\leq 10^{-8} \mathrm{M}: \mathrm{a} 1 \mathrm{a}-\mathrm{b}, \mathrm{b}-\mathrm{d}$ - adrenoceptors & & ADRA1A, ADRA1B, ADRA1D \\
\hline & $\leq 10^{-8} \mathrm{M}: \mathrm{a} 2 \mathrm{2a}-\mathrm{b}$-, c-adrenoceptors & & $A D R A 2 A, A D R A 2 B, A D R A 2 C$ \\
\hline & $\geq 10^{-6} \mathrm{M}: \beta 1-, \beta 2-, \beta 3$-adrenoceptors & & $A D R B 1, A D R B 2, A D R B 3$ \\
\hline \multicolumn{4}{|l|}{ Peptidergic } \\
\hline Vasoactive intestinal peptide (VIP) & VIP-1, VIP-2, and VIP/PACAP receptors & VIP & VIPR1, VIPR2 \\
\hline
\end{tabular}

PACAP, pituitary adenylate cyclase-activating polypeptide.

[24]. This observation implies important functions of sensory nerves for regulating chondrogenic differentiation during limb growth in embryonic development. In line with this, Edoff and colleagues [22,25] reported that articular chondrocytes respond specifically to the application of CGRP by increasing the cAMP level. They assume that dorsal root ganglion neurons which project to growth plate cartilage may influence chondrocyte differentiation via CGRP. It is described that increased levels of cAMP suppress terminal differentiation of chondrocytes and matrix mineralization [26] which makes it likely that local release of CGRP can delay chondrocyte hypertrophy and subsequent terminal differentiation through modulating cAMP level (Figure 1). Whereas sympathetic nerve fibers have been abundantly localized to subchondral bone marrow [27] and synovial joint tissues [28], no reports exist about close contact to chondrocytes or innervation of healthy articular cartilage.

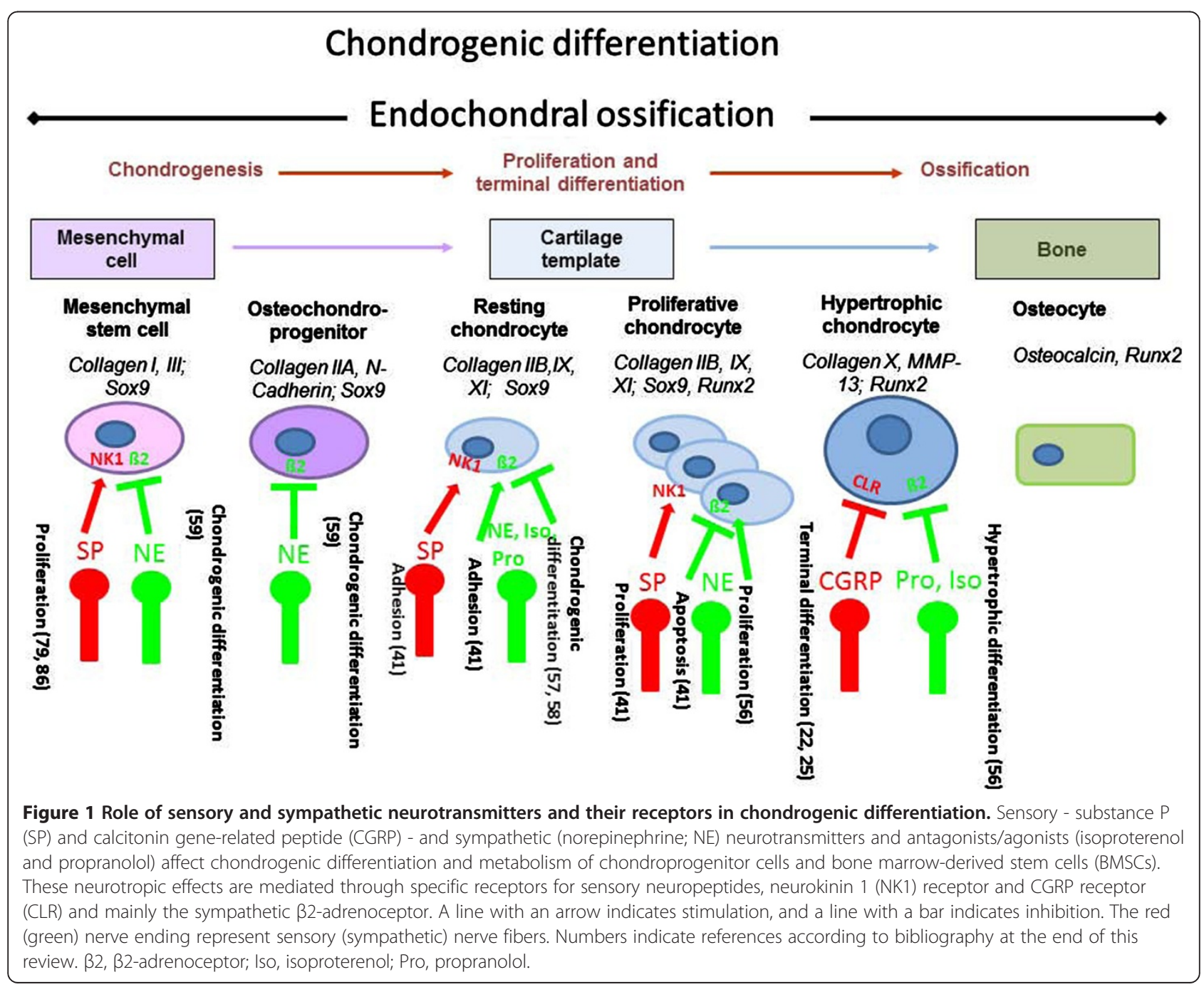




\section{Sensory and sympathetic nerve fibers in osteoarthritis}

OA, a degenerative disorder of diathrodial joints culminating in the irreversible destruction of the articular cartilage, is clinical highly relevant and a burden for the health-care system and society because of high costs for diagnosis, treatment, sick leave, rehabilitation, and early retirement [29]. However, OA is not exclusively a disorder of articular cartilage; it can be considered an organ failure of the whole joint with additional abnormalities especially in subchondral bone, ligaments, synovium, and the joint capsule. Suri and colleagues [30] have localized both sensory (SP- and CGRP-positive) and sympathetic (NPY-positive) nerve fibers in similar distributions to the articular cartilage in human tibiofemoral OA. In the pathogenesis of $\mathrm{OA}$, nerves grow into joint structures through vascular channels mainly from subchondral bone breaching through the tidemark. The exclusively perivascular localization of nerves in the articular cartilage implies that vascularization is the driving force behind its innervation. Notably, vascularization of the non-calcified cartilage was found throughout a wide range of histological OA stages and was not restricted to end-stage OA. Possibly, scoring of nervous innervation and vascularization of cartilage might be exploited as a measure for severity of degradative changes in OA pathogenesis. The authors suggest that innervation of the articular cartilage is therefore a potential source of pain in patients with knee OA. These authors and others have also shown sympathetic and perivascular sensory innervation of tibial osteophytes, the latter localized to the base of osteophytes [30,31]. Sensory innervation of osteophytes may explain why radiological grading of osteophytosis is associated with reported pain severity [32]. However, despite the clinical importance of pain in OA, pain mechanisms are poorly understood. It is unclear which structures in the joint give rise to OA pain, and the nature of OA pain (nociceptive versus neuropathic) is a matter of ongoing discussion [33].

In line with Suri and colleagues [30], it was recently demonstrated that there was a marked preponderance of sensory over sympathetic nerve fibers in ankle bone and synovial tissue in patients with OA compared with patients with anterior knee pain after primary arthroplasty [34]. In patients with chronic RA and end-stage OA, this study of synovial tissues demonstrated a preponderance of SP-positive nerve fibers relative to CGRP-positive nerve fibers in patients with RA but not in patients with end-stage OA. Here, an even balance exists between SPpositive and CGRP-positive nerve fibers. Finally, the authors observed a positive correlation between the density of CGRP-positive and sympathetic nerve fibers in OA, which they interpreted as an anti-inflammatory signal. Such a positive correlation was not observed in synovial tissue of patients with RA, which demonstrates a markedly reduced presence of sympathetic nerve fibers. Thus, in the less-inflamed tissue of patients with OA, coupling of two anti-inflammatory pathways might dampen the inflammatory process, a situation that is lost in the tissue of patients with RA, indicating a neuroendocrine uncoupling mechanism [9]. A drastic loss of specifically sympathetic nerve fibers in synovial joint tissues of patients with RA has previously been demonstrated [35]; however, it appears that during OA pathogenesis this does not happen. Instead, inflammatory changes in synovium of OA joints are associated with a massive destruction of the total capillary and neuronal, sympathetic and sensory nervous network which is present in normal synovium [36]. Notably, in a murine OA model, it was suggested that in some soft joint tissues, CGRP- and SP-positive nerve fibers disappeared 5 weeks after induction of $\mathrm{OA}$ with intra-articular injection of collagenase [37]. This quite aggressive method of OA induction leads to profound degenerative alterations in joint tissue after a few weeks, comparable with late-stage $\mathrm{OA}$ in humans. The mechanisms which destruct the normal vascular and neural network are not identified yet, and also it is unclear whether nerves are destroyed as a consequence of OA or whether pathogenesis of $\mathrm{OA}$ is facilitated because of nerve disappearance. A study by Salo and colleagues [10] indicated that loss of SP- and CGRP-positive joint innervation always preceded histological changes of cartilage degeneration. They used a mice model which usually developed a mild form of OA, but surgical ablation of joint innervation caused the development of severe patellofemoral OA. Their findings would be consistent with the hypothesis that an age-related loss of joint innervation may contribute to the development of OA. Whether this is comparable to the human situation remains to be investigated, and insight into these mechanisms will require reproducible OA animal models resembling slow proceeding pathogenesis of human $\mathrm{OA}$ and enabling longitudinal studies from early onset of the disease on to late stages.

\section{Nerve repellent factors in cartilage}

Why it is that healthy and also mostly diseased articular cartilage is basically not innervated is still not sufficiently clarified. Possibly, the lack of blood vessels prevents innervation or vice versa. Trying to find underlying molecular mechanisms for this unique phenomenon suggests the presence of specific axon guidance or nerve repellent factors in cartilage. Well known sensory and sympathetic nerve-specific axon guidance molecules or repellent factors belong to the semaphorin family. A selective repellent of SP- and CGRP-positive sensory nerve fibers is semaphorin (Sema)3A, which, besides being expressed in neuronal tissues, is expressed in developing cartilage and bone [38], in intervertebral discs [39] and recently was even found in adult articular cartilage [40]. Gomez and colleagues [38] 
showed convincingly that the Sema signaling system consisting of Sema3A and its receptors plexin (Plx)-A3/neuropilin (NP)-1 is expressed in resting, pre-hypertrophic, and hypertrophic chondrocytes in growth cartilage before the onset of neurovascular invasion during endochondral ossification. They suggest that the Sema3A/Plx-A3/NP-1 pathway would inhibit neuro-vascularization of the cartilage anlage early in skeletal development. This block could be removed later and locally by the downregulation of key receptor chains, the elimination of Sema3A-producing cells (for example, hypertrophic chondrocytes), or by the expression of competitors of Sema3A (such as vascular endothelial growth factor (VEGF) ${ }_{165}$ ), for instance, by perichondral cells and hypertrophic chondrocytes. Inhibition of Sema3A signaling would then allow blood vessel and nerve fiber invasion of the diaphysis and thus the onset of endochondral ossification together with the start of local neural or vascular regulation (or both) of bone formation. Later, during the ossification process, Sema3A may provide a repulsive guidance, allowing the growing nerve fibers to be directed to their targets in bone tissue. Another group reported that gene and protein expression of Sema3A and its receptor NP-1 was significantly elevated in chondrocytes from OA cartilage as compared with chondrocytes from normal cartilage and that the Sema3A expression is closely correlated with chondrocyte cloning, which is a characteristic feature of OA cartilage [40]. The authors imply the possibility that Sema3A plays a role in the pathogenesis of chondrocyte cloning through antagonizing and inhibition of VEGF-mediated cell migration. All together, these studies assign a novel function to Sema in chondrogenic differentiation during embryonic development and cartilage degeneration in adults in addition to their known axon guidance role.

\section{Sensory and sympathetic neurotransmitters and their receptors expressed by chondrocytes}

Besides their classic function in nociception, SP and CGRP appear to have extra functions in the musculoskeletal system. Lately, murine costal and human articular chondrocytes have been recognized to endogenously produce SP and its receptor NK1 [41,42]. Earlier, SP was immunolocalized to articular cartilage of dog shoulder joints. Expression and localization were increased in chondrocytes and within the extracellular matrix after lowimpact regimented exercise, indicating a role in signaling pathways through which chondrocytes respond to mechanical stimulation [43]. This was indeed demonstrated by Millward-Sadler and colleagues [44], who suggested that $\mathrm{SP}$ is involved in mechanotransduction via the NK1 receptor. They found SP to be necessary for a hyperpolarization response of the cell membrane, and concomitant changes in gene expression as a response to mechanical stimulation indicate a role of SP in the maintenance of articular cartilage matrix integrity and function. The same group demonstrated that normal and OA chondrocytes reacted differently to mechanical stimulation in that OA chondrocytes upregulated gene expression of the SP encoding gene, Tac1, whereas non-OA chondrocytes did not respond with changes in Tac1 gene expression [45]. In addition, we recently demonstrated that costal chondrocytes from newborn mice when stimulated with SP induced proliferation and cell-matrix adherence by stimulating formation of focal adhesion contacts. These effects are mediated specifically through the NK1 receptor [41]. Our observation implies that SP might modulate proliferation rate of growth plate chondrocytes and consequently terminal differentiation during endochondral ossification. It is thus conceivable that, in chondrocyte physiology and in chondrogenic differentiation during skeletal growth, endogenous SP production acts as a trophic, anabolic factor and does not function as a classic neuropeptide (Figure 1 and Table 3). However, in adults, the detection of higher levels of SP in synovial fluid from patients with RA and OA, and increased expression of NK1R, indicates catabolic effects of SP on articular cartilage [46]. In addition, transforming growth factor-beta and basic fibroblast growth factor play an important role as inductor or promoter for production of SP in synovial fibroblasts. These data are supported by Im and colleagues [47], who elegantly demonstrated that SP induces interleukin 1-beta (IL-1 $\beta$ ) release. They propose a mechanism by which basic fibroblast growth factor, together with SP, reduces proteoglycan deposition and stimulates production and release of matrix metalloprotease (MMP)-13 in human articular chondrocytes and thus accelerates catabolic processes in cartilage. All together, these observations suggest that SP has autocrine functions and can modulate chondrocyte metabolism and cartilage homeostasis differentially during skeletal growth and in pathophysiology. As in synovial cells where SP is described as potent mediator of inflammation by promoting secretion of prostaglandin E2, several MMPs [48], reactive oxygen species [49], IL-1, and tumor necrosis factor-alpha [50], SP also seems to act in a catabolic way in chondrocytes and to promote cartilage degradation. To date, there are no reports listed in Pubmed with respect to production of CGRP and its receptors in cartilage. As in bone metabolism where CGRP is described as an anabolic factor by stimulating osteoblast activity and thus bone formation [51,52], one might hypothesize that CGRP has similar anabolic effects in cartilage physiology.

Notably, compared with trauma patients, OA patients have lower VIP concentration in synovial fluid [53]. VIP has been shown to predominantly possess an antiinflammatory action (reviewed in [54]); it is very efficient at ameliorating the pathology of several models of 
Table 3 Sensory and sympathetic receptors and neurotransmitters expressed in cells and tissues of diathrodial joints

Receptors and tissue (cells)
NK1R
Chondrocytes of articular cartilage
Chondrocytes of costal cartilage
Proliferating and hypertrophic
chondrocytes of the growth plate
Chondrocytes of OA cartilage
Chondrocytes from fracture callus
Osteoblasts
Osteoclasts and precursors (bone
marrow-derived macrophages)
BMSCs
Synovial fibroblasts
CL receptor/RAMP
Osteoblasts
Osteoclasts
BMSCs

\section{$\beta$-Adrenoceptors}

Chondrocytes of articular cartilage (B2-AR)

Chondrocytes of costal cartilage ( $\beta 2-A R$ )

Chondrocytes of growth plates (all zones) ( $\beta 2-A R$ )

Chondrocytes of OA cartilage (B2-AR)

Chondrogenic progenitor cells from $O A$ cartilage ( $\beta 2-A R$, in vitro)

Osteoblasts ( $\beta 2-A R)$

Osteoclasts and precursors (bone marrow-derived macrophages)

BMSCs ( $\beta 2 / \beta 3-A R)$

Synovial cells

\section{a1-Adrenoceptors}

Chondrocytes of OA articular cartilage

Chondrocytes in fracture callus

Mesenchymal cells in fracture callus
Neurotransmitters and tissue (cells)

Substance $P$

Chondrocytes of articular cartilage

Chondrocytes of costal cartilage

Proliferating and hypertrophic chondrocytes of the growth plate

Chondrocytes of OA cartilage

Chondrocytes from fracture callus

BMSCS

Synovial fibroblasts

Synovial fluid

Calcitonin gene-related peptide

Osteoblasts

BMSCS

Synovial fluid

Tyrosine-hydroxylase (rate-limiting enzyme for catecholamine synthesis)

Chondrocytes of the cartilage deep zone

Costal chondrocytes

Hypertrophic chondrocytes of the growth plate

Osteoblasts

Osteoclasts

Proliferating and chondrogenic/ differentiating BMSCs in vitro

Synovial cells during RA pathogenesis

Synovial fluid
Table 3 Sensory and sympathetic receptors and neurotransmitters expressed in cells and tissues of diathrodial joints (Continued)

\section{a2-Adrenoceptors}

Chondrocytes of articular cartilage

Chondrocytes of the growth plate

(different zones)

Chondrocytes of fracture callus

Mesenchymal cells of fracture callus

Osteoblasts

Osteoclasts and precursors (bone marrow-derived macrophages)

Synovial cells

VIP receptors

VIP

Osteoclasts (VIP-1/-2) and PACAP

Synovial fluid

Osteoblasts (VIP-1/-2)

OA cartilage (?)

Bone marrow-derived macrophages (PACAP)

Synovial cells (VIP-1/-2)

AR, adrenoceptor; BMSC, bone marrow-derived stem cell; $\mathrm{CL}$, calcitonin gene-related peptide; NK1R, neurokinin 1 receptor; OA, osteoarthritis; PACAP, pituitary adenylate cyclase-activating polypeptide; RA, rheumatoid arthritis; RAMP, receptor activity-modifying protein; VIP, vasoactive intestinal peptide.

autoimmune disorders, including RA. Delgado and colleagues [55] showed that treatment with VIP significantly reduced the incidence and severity of collagen-induced arthritis, abrogating joint swelling and the destruction of cartilage and bone. These therapeutic effects were associated with the downregulation of both inflammatory and autoimmune components of the disease [55]. It might be suggested that VIP concentration in synovial fluid is negatively associated with progressive joint damage in OA and has potential as an indicator of disease severity.

Not much is known about production of sympathetic neurotransmitters and their receptors in chondrocytes. $\beta 2$ - and $\alpha 2 a-A R s$ have been found on growth plate chondrocytes at different developmental stages [56-58], and expression of $\beta 2$ - and $\alpha 1 / \alpha 2$-ARs was detected in neonatal murine costal chondrocytes [41] and in adult human articular OA chondrocytes [45] (Table 3 and Figure 1). In general, it appears that signaling through $\beta 2$-ARs interferes with chondrogenic differentiation by inhibiting collagen II, collagen X, and Indian hedgehog expression in part through repression of sox6 and sox9 signaling [56-58]. In addition, we demonstrated that signaling through $\beta 2$-ARs inhibits apoptosis of murine costal chondrocytes and increases cell-matrix adhesion in vitro [41]. In this line, Lai and Mitchell [56] showed that signaling through $\beta 2$-ARs stimulates murine growth plate chondrocyte proliferation and inhibits terminal differentiation. All together, these sparse data on sympathetic effects in chondrocytes imply 
that signaling through $\beta$-ARs interferes with chondrogenic differentiation by suppressing production of important chondrogenic matrix molecules (Figure 1). This note is corroborated by recent work from Jenei-Lanzl and colleagues [59], who clearly showed that NE stimulation of human adult chondroprogenitor cells inhibits collagen II and glycosaminoglycan production and accelerates the hypertrophic pathway by induction of collagen $\mathrm{X}$ and MMP-13 expression, which is opposite to the above reports. In this study, no change in proliferative activity of progenitor cells was observed. This might be because, in the above studies, growth plate chondrocytes were analyzed reflecting an embryonic chondrogenic differentiation status whereas Jenei-Lanzl and colleagues [59] investigated chondrogenic differentiation of adult mesenchymal stem cells and chondrogenic progenitor cells from $\mathrm{OA}$ cartilage. In line with this, $\mathrm{Li}$ and colleagues [60] showed that $\beta 2 / 3$-AR gene and protein expression is increased during adipogenic differentiation of murine bone marrow-derived stem cells (BMSCs). Application of $\beta$-AR antagonists positively affects adipogenic differentiation, whereas agonists suppress adipogenic differentiation of BMSCs by downregulating adipogenic marker genes. The authors suggest that these effects are mediated by increased intracellular cAMP level which activates the PKA pathway which presumably mediates downregulation of two key adipogenic transcription factors: $\mathrm{C} / \mathrm{EBP} \alpha$ and $\mathrm{C} / \mathrm{EBP} \beta$.

In conclusion, one can speculate that sympathetic neurotransmitters (for example, NE) alter chondrogenic differentiation of chondrogenic progenitor cells by inhibiting chondrocyte hypertrophy via $\beta$-ARs during embryonic endochondral ossification (Figure 1 and Table 3). In adults, NE might reduce the self-regeneration capacity of articular cartilage by accelerating the hypertrophic pathway and thus play a role in development and manifestation of OA. In addition, signaling through $\beta$-AR inhibits adipogenic differentiation of BMSCs which might be important in the pathogenesis of obesity and osteoporosis in adults.

\section{Sensory and sympathetic nerve fiber innervation in bone tissues}

Several reports have demonstrated an intensive network of sensory and sympathetic nerve fibers within the skeleton, not only in the periosteum but also within trabecular and less in cortical bone, bone marrow, and epiphyseal growth plate [19,23,61-63]. Many of those nerve fibers are associated with blood vessels, but several blood vessel-unrelated nerves and free nerve endings have also been observed. Besides the possibility that sensory and sympathetic nerve fibers have important roles in skeletal pain transmission, accumulating evidence suggests that sensory and sympathetic nerve fibers do have a role in bone remodeling and osteogenic differentiation of precursor cells during skeletal growth. In bone, the areas with the highest metabolic activity receive the richest sensory and sympathetic innervation [64]. This is in line with a study by Offley and colleagues [11], who used selective lesioning of the unmyelinated sensory neural pathway to determine the role of capsaicinsensitive sensory SP- and CGRP-containing afferents in the maintenance of normal bone balance in skeletally mature rats. Collectively, their results indicate that capsaicin-sensitive sensory neurons contribute to skeletal homeostasis and that lesioning these neurons caused enhanced bone resorption, a reduction in new bone formation, a subsequent loss of trabecular connectivity and thickness, and ultimately an increase in bone fragility [11].

Very importantly, bone cells express receptors for many of the neuronal messengers present in these skeletal nerve fibers, and activation of such receptors leads to profound effects on the activity of both osteoblasts and osteoclasts, strongly suggesting the existence of neuro-osteogenic or neuro-immuno-osteogenic interactions (Table 3 and Figure 2).

Experimental studies have provided accumulating evidence that peripheral nerve fibers not only are important in normal bone homeostasis and skeletal growth but also influence repair mechanism after bone trauma (for example, fracture healing). Aro [65] demonstrated that, in denervated limbs, fracture callus size was clearly reduced at a later stage of the healing process. Other studies demonstrated larger callus formation after nerve resection $[7,66]$ and corroborate the observation that bone union is faster and fracture callus volume is increased in patients with head injuries involving coma without clarifying whether this is neuronal, mediated as a direct consequence of the head injury or metabolical, or biochemical as an indirect consequence [1]. At early time points after fracture, TH-, CGRP-, and SP-positive fibers grow into the callus prior to vascularization whereas at later time points these nerve fibers retract to the periosteum $[62,67]$. Li and colleagues [68] elegantly demonstrated in an angular rat fracture model that innervation of CGRP-positive nerves is higher at the concave compared with the convex site of the fracture. The site-specific changes in CGRP innervation correlate with the amount of bone formation on both the convex and concave sides of angular fractures. Given that the concave side of the angular fracture requires more bone formation than the convex to correct the deformity and that CGRP was clearly more abundant on the concave side, it is reasonable to assume that the peripheral sensory nervous system plays an important role in local bone turnover and that a restored nerve supply could be essential for normal bone regeneration during fracture healing. 




VIP has been demonstrated to play an important role in the control of osteoclast formation because destruction of nerves expressing VIP by guanethidine treatment increases the number of osteoclasts [69]. The underlying mechanisms hint to inhibition of osteoclast formation by inhibiting the stimulatory effect of vitamin D3 $(1,25(\mathrm{OH})$ 2D3) [70]. This inhibition of osteoclast formation through VIP is mediated via its inhibiting effect on vitamin D3induced upregulation of receptor activator of nuclear factor kappa-B (RANK) and its ligand (RANKL) and by counteracting a decrease of osteoprotegerin (OPG) caused by vitamin D3 (Figure 2). Thus, VIP would be an excellent candidate that might influence inflammatory processes. Typically, sympathetic nerve fibers carry the two markers $\mathrm{TH}$ and NPY, which are important determinants of the catecholaminergic phenotype [71]. However, this can change because there likely exists a bidirectional communication between sympathetic nerve fibers and mesenchymal tissue which influences the neurotransmitter phenotype. For example, periosteal tissue can change the neurotransmitter phenotype of sympathetic nerve fibers [72]. The contact of periosteal cells with sympathetic nerve fibers changes the catecholamine phenotype, leading to a peptidergic and cholinergic phenotype (VIP and acetylcholine instead of NE). It is suggested that several cytokines of the gp130 family and neurotrophic factors determine the switch [73]. During development or under inflammatory conditions such as in arthritis or OA, sympathetic innervation of bone might change, leading to completely different control of bone homeostasis. 
An interesting note concerns the involvement of the sympathetic nervous system in the mechanism of bone loss in long-term microgravity in space [74]. The authors suggest that an exposure to prolonged microgravity may enhance sympathetic neural traffic not only to muscle but also to bone. This sympathetic enhancement increases plasma NE level, inhibits osteogenesis, and facilitates bone resorption through $\beta$-ARs signaling which in combination leads to reduced bone mass. They suggest that the use of $\beta$-AR blockers to prevent bone loss in microgravity may be reasonable, which is in line with suggestions that $\beta$ blockers may reduce bone loss and fracture risk in postmenopausal women [12]. On the other hand, Sherman and Chole [75] report that low NE concentrations which are observed after chemical sympathectomy induce bone resorption and decrease bone formation. Therefore, there is not an adequate evidence base to support using $\beta$-blockers as a treatment for osteoporosis, nor can $\beta$-blockers be regarded as a discriminating risk factor for fracture assessment. Until there are definitive randomized controlled trials with $\beta$-blockers, which include fracture as an endpoint, it is unlikely that the current confusing situation will be resolved [12].

Sensory innervation in bone also might play a role in degenerative musculoskeletal disorders as in OA. In diathrodial joints, the subchondral bone contains sensory nerve fibers [76]; however, subchondral regions of normal knee joints are sparsely innervated by SP- and CGRP-nociceptive fibers [77]. SP- and CGRP-positive nerve fibers were identified in the subchondral bone of patients with OA, but it is still unclear whether both sensory nerve fiber types display increased sensitivity or number in OA joints [37]. Both types of nerve fibers have been localized in osteophytes, and perivascular SPpositive nerve fibers were found at the base of osteophytes in horse metacarpophalangeal OA [30,31]. In addition to SP-positive sensory nerve fibers, cells resident in cystic lesions (that is, vascular channels) of the subchondral bone plate of OA knee joints stained positive for SP itself [78]; however, the nature of these cells could not be identified. This note is supported by the observation that SP protein staining in areas of remodeling and in erosion channels of the subchondral bone is increased in OA pathogenesis but no respective nerve fibers were identified [79]. How changes in sensory and sympathetic joint innervation and their respective neurotransmitters contribute to abnormal subchondral bone remodeling and osteophyte formation during the pathogenesis of OA is mostly unknown.

\section{Sensory neuropeptides and catecholamines and their receptors produced in bone}

There is accumulating evidence that sensory and sympathetic neurotransmitters such as SP, $\alpha$ CGRP, VIP, and
NE have crucial trophic effects which are essential for proper bone metabolism and bone remodeling. In the recent literature, some comprehensive reviews comment on expression of $\alpha$ CGRP, SP, and sympathetic catecholaminergic (NE) and peptidergic (VIP) neurotransmitters as well as the presence of their receptors on osteocytes, osteoblasts, osteoclasts, bone marrow-derived macrophages, and BMSCs and their contribution to regulation of osteogenic differentiation, osteoclastogenesis, and consequently bone resorption and bone formation in physiological and pathophysiological situations $[19,51,54,80-82]$ (Figure 2 and Table 3).

With respect to the sympathetic nervous system, most cited articles report about the regulation of bone remodeling in adults through alteration in post-synaptic $\beta$-AR signaling $[51,82]$. A recent article by Ma and colleagues [83] addressed the contribution of endogenous sympathetic signaling and NE homeostasis to the control of bone remodeling. They convincingly demonstrated that differentiated osteoblasts, like sympathetic presynaptic neurons, can transport and catabolize NE and thus may contribute to NE clearance within the richly vascularized bone marrow microenvironment. Their findings indicate that the control of NE reuptake by the norepinephrine transporter is an integral part of the homeostatic system whereby bone remodeling is regulated. These data point to the existence of multiple endogenous regulatory pathways modulating bone remodeling via the control of both NE release and NE clearance. Lastly, the authors suggest that drugs blocking norepinephrine transporter activity, which are used for the treatment of depression and attention deficit hyperactivity disorder, may have a deleterious effect on the skeleton.

With respect to sensory neuropeptides, $\alpha$ CGRP-deficient mice suffer from osteopenia [52], and this clearly shows that CGRP can be considered a bone anabolic factor. The osteopenic phenotype was caused by a significant reduction of bone formation; however, osteoblast numbers were not affected, which suggests that CGRP regulates mainly functional activity of osteoblasts and not their mitotic activity. In addition, in mouse bone marrow cultures stimulated to generate osteoclasts by $1,25(\mathrm{OH})$ 2D3, CGRP dose-dependently decreased the numbers of tartrate-resistant alkaline phosphatase-positive multinucleated cells [84]. These data need to be considered together with the notion that osteoporosis is a significant complication of stroke as frequently patients who survive an acute stroke face hip fractures. These strokerelated complications can be attributed to reduced bone mass due to increase in osteoclast-mediated bone resorption and subsequent decreased osteoblast-mediated bone formation (reviewed in [3]).

Notably, a recent study aimed to analyze the effect of CGRP on adipogenic differentiation of BMSCs [85]. The 
authors demonstrated that simultaneous downregulation of peroxisome proliferator activated receptor-gamma and upregulation of CGRP in rabbit BMSCs efficiently suppressed alcohol-induced adipogenic differentiation while promoting osteogenic differentiation. This is an important observation with respect to development of alcohol-induced osteonecrosis of the femoral head and might have implications for future treatment strategies. Whereas CGRP is a priori characterized as a bone anabolic factor [51], SP has both bone resorbing and bone formation activities. Some studies reported opposite effects on bone formation for SP depending on its concentration. Whereas SP concentrations of more than $10^{-8} \mathrm{M}$ stimulate osteoblast differentiation and bone matrix mineralization [86,87], SP concentrations of less than $10^{-8} \mathrm{M}$ block osteogenic differentiation of rat BMSCs but induce proliferation and general protein synthesis [88]. In addition, SP stimulates proliferation of mesenchymal precursor cells and other cells (that is, chondrocytes in a concentration-dependent manner). By blocking the NK1R chemically in rats for 2 weeks, Kingery and colleagues [89] reported a significant reduction in tibial and femoral cancellous bone mineral density as observed in osteoporosis. This strongly suggests a role for SP in maintaining bone integrity and regulation of bone formation and bone resorption. Another observation indicates that ovariectomy which leads to reduced levels of SP at the fracture site was accompanied by impaired mechanical bone properties [90]. These reports indicate a positive effect of SP on bone formation if high concentrations of SP are available and a negative effect if SP concentration is low or if the neuropeptide is absent (Figure 2). In line with these data, our group observed that absence of SP reduces pain sensitivity and mechanical stability of bone in general and negatively impacts on bone structure in an adult murine model of endochondral ossification [67]. The micro-architecture of cortical bone is impaired in SP-deficient mice (Tachykinin 1 gene-deficient), and the absence of SP reduces bone formation rate indicated by lower bone mass and mineralization. Notably, chemical destruction of the sympathetic nervous system with 6hydroxy dopamine has even more pronounced effects on bone architecture and bone remodeling by inducing bone resorption without affecting bone formation.

The signaling pathways through which VIP, SP, and CGRP effects on chondro-osteogenic differentiation or osteoclastogenesis are mediated remain largely unknown. Like CGRP, VIP suppresses bone resorptive activities through regulation of RANKL/OPG expression [70]. Yoo and colleagues [91] demonstrated that a decrease in RANKL/OPG ratio was comparable to CGRP/VIP treatment of a pre-osteoblastic cell line either in combination with sheer stress application or separately. They conclude that mechanical stress and VIP/CGRP regulate bone resorptive activities in a similar cellular signal transduction pathway. VIP effects on RANKL and OPG are mediated by an increase in CAMP, indicating that VIP stimulates the PKA/CREB pathway in mouse calvarial osteoblasts, bone marrow, and stromal cells $[92,93]$. However, RANKL mRNA expression in mouse calvarial osteoblasts can also be mediated by the MEK/ ERK pathway [94] and this is in line with other reports demonstrating involvement of MEK/ERK in intracellular signaling by VIP [95]. A recent article [96] presents strong evidence that pro-osteogenic differentiation effects of SP are mediated via activating the $\mathrm{Wnt} / \beta$-catenin signaling pathway. The same group demonstrates that SP induces proliferation of BMSCs through activation of this pathway [97]. Also, CGRP appears to mediate its effects on osteoblasts through the Wnt/ $\beta$-catenin pathway [98]. CGRP presumably exerts its anabolic action on bone cells by stimulating canonical Wnt signaling through stabilization of $\beta$-catenin and by inhibiting osteoblast apoptosis, thus favoring local bone formation.

All together, these data imply that sensory and sympathetic neurotransmitters have crucial trophic effects which are critical for proper osteogenic differentiation and bone metabolism during embryonic skeletal growth and bone regeneration in adults (that is, after fracture) in addition to their classic neurological actions.

\section{Conclusions}

Sensory and sympathetic nerves and their neurotransmitters are important neuronal effectors regulating bone and cartilage physiology and playing decisive roles in musculoskeletal pathophysiology. Notably, many resident cells of the osteoarticular system contain receptors for sympathetic and sensory neurotransmitters and thus can respond to their stimuli. During endochondral ossification, sensory neuropeptide SP promotes proliferation of stem cells and growth plate chondrocytes, whereas signaling through $\beta$-ARs inhibits chondrogenic differentiation of osteo-chondroprogenitor cells and terminal differentiation of chondrocytes. In bone metabolism and bone remodeling, CGRP and VIP have anabolic effects, inducing osteoblast activity and inhibiting osteoclastogenesis, whereas SP also has catabolic effects depending on its concentration. Effects of the sympathetic nervous system on bone homeostasis are complex; however, it is discussed that blocking signaling through $\beta$-ARs can prevent bone loss, suggesting that high catecholamine concentrations shift the balance toward bone resorption by inhibiting osteoblast differentiation. During pathogenesis of OA, sympathetic and sensory nerve fibers grow into cartilage from subchondral bone. In late-stage OA synovial tissue, there are more sensory nerves compared with sympathetic nerve fibers; however, it appears that both types of nerves 
become destroyed during progression of OA. In light of all data, it is more and more evident that sensory and sympathetic nerves and their neurotransmitters critically affect bone and cartilage physiology and are crucially involved in musculoskeletal disorders.

Note: This article is part of the series 'At the interface between
immunology and neurology in rheumatic diseases', edited by
Rainer Straub. Other articles in this series can be found at
http://arthritis-research.com/series/neurology.

\section{Abbreviations}

AR: adrenoceptor; BMSC: bone marrow-derived stem cell; CGRP: calcitonin gene-related peptide; IL: interleukin; MMP: matrix metalloprotease;

NE: norepinephrine; NP: neuropilin; NPY: neuropeptide Y; OA: osteoarthritis; OPG: osteoprotegerin; PIx: plexin; RA: rheumatoid arthritis; RANKL: receptor activator of nuclear factor kappa-B ligand; Sema: semaphorin; SP: substance P; TH: tyrosine-hydroxylase; VEGF: vascular endothelial growth factor; VIP: vasoactive intestinal peptide.

\section{Acknowledgments}

The author thanks Rainer Straub for critically revising this manuscript. This work was supported by a grant from BMBF (Federal Ministry of Education and Research, Bonn, Germany) to SG and RS (AZ. 01EC1004D, subproject 5, consortium Immunopain).

Published online: 22 November 2014

\section{References}

1. Perkins R, Skirving AP: Callus formation and the rate of healing of femoral fractures in patients with head injuries. J Bone Joint Surg Br 1987, 69:521-524.

2. Dauty M, Perrouin Verbe B, Maugars Y, Dubois C, Mathe JF: Supralesional and sublesional bone mineral density in spinal cord-injured patients. Bone 2000, 27:305-309.

3. Poole KE, Reeve J, Warburton EA: Falls, fractures, and osteoporosis after stroke: time to think about protection? Stroke 2002, 33:1432-1436.

4. Jones KB, Mollano AV, Morcuende JA, Cooper RR, Saltzman CL: Bone and brain: a review of neural, hormonal, and musculoskeletal connections. lowa Orthop J 2004, 24:123-132.

5. Garcia-Castellano JM, Diaz-Herrera P, Morcuende JA: Is bone a target-tissue for the nervous system? New advances on the understanding of their interactions. lowa Orthop J 2000, 20:49-58.

6. Hukkanen M, Konttinen YT, Rees RG, Santavirta S, Terenghi G, Polak JM: Distribution of nerve endings and sensory neuropeptides in rat synovium, meniscus and bone. Int J Tissue React 1992, 14:1-10.

7. Madsen JE, Hukkanen M, Aune AK, Basran I, Moller JF, Polak JM, Nordsletten L: Fracture healing and callus innervation after peripheral nerve resection in rats. Clin Orthop Relat Res 1998, 230-240.

8. Gajda M, Adriaensen D, Cichocki T: Development of the innervation of long bones: expression of the growth-associated protein 43. Folia Histochem Cytobiol 2000, 38:103-110.

9. Dirmeier M, Capellino S, Schubert T, Angele P, Anders S, Straub RH: Lower density of synovial nerve fibres positive for calcitonin gene-related peptide relative to substance $P$ in rheumatoid arthritis but not in osteoarthritis. Rheumatology (Oxford) 2008, 47:36-40.

10. Salo PT, Seeratten RA, Erwin WM, Bray RC: Evidence for a neuropathic contribution to the development of spontaneous knee osteoarthrosis in a mouse model. Acta Orthop Scand 2002, 73:77-84.

11. Offley SC, Guo TZ, Wei T, Clark JD, Vogel H, Lindsey DP, Jacobs CR, Yao W, Lane NE, Kingery WS: Capsaicin-sensitive sensory neurons contribute to the maintenance of trabecular bone integrity. J Bone Miner Res 2005, 20:257-267.
12. Reid IR: Effects of beta-blockers on fracture risk. J Musculoskelet Neuronal Interact 2008, 8:105-110.

13. Zhang Y, Lu L, Furlonger C, Wu GE, Paige CJ: Hemokinin is a hematopoietic-specific tachykinin that regulates B lymphopoiesis. Nat Immunol 2000, 1:392-397.

14. Maggi CA: The mammalian tachykinin receptors. Gen Pharmacol 1995, 26:911-944.

15. Hartung HP, Toyka KV: Activation of macrophages by substance P: induction of oxidative burst and thromboxane release. Eur J Pharmacol 1983, 89:301-305.

16. Rosenfeld MG, Mermod JJ, Amara SG, Swanson LW, Sawchenko PE, Rivier J, Vale WW, Evans RM: Production of a novel neuropeptide encoded by the calcitonin gene via tissue-specific RNA processing. Nature 1983, 304:129-135

17. Poyner DR, Sexton PM, Marshall I, Smith DM, Quirion R, Born W, Muff R, Fischer JA, Foord SM: International Union of Pharmacology. XXXII. The mammalian calcitonin gene-related peptides, adrenomedullin, amylin, and calcitonin receptors. Pharmacol Rev 2002, 54:233-246.

18. Molinoff PB: Alpha- and beta-adrenergic receptor subtypes properties, distribution and regulation. Drugs 1984, 28:1-15.

19. Lerner UH, Persson E: Osteotropic effects by the neuropeptides calcitonin gene-related peptide, substance $P$ and vasoactive intestinal peptide. J Musculoskelet Neuronal Interact 2008, 8:154-165.

20. Poole AR, Kojima T, Yasuda T, Mwale F, Kobayashi M, Laverty S: Composition and structure of articular cartilage: a template for tissue repair. Clin Orthop Relat Res 2001, 391 Suppl:S26-S33.

21. Schwab W, Funk RH: Innervation pattern of different cartilaginous tissues in the rat. Acta Anat (Basel) 1998, 163:184-190.

22. Edoff $K$, Grenegard M, Hildebrand C: Retrograde tracing and neuropeptide immunohistochemistry of sensory neurones projecting to the cartilaginous distal femoral epiphysis of young rats. Cell Tissue Res 2000, 299:193-200.

23. Hara-Irie F, Amizuka N, Ozawa H: Immunohistochemical and ultrastructural localization of CGRP-positive nerve fibers at the epiphyseal trabecules facing the growth plate of rat femurs. Bone 1996, 18:29-39.

24. Oliva F, Tarantino U, Maffulli N: Immunohistochemical localization of calcitonin gene-related peptide and substance $P$ in the rat knee cartilage at birth. Physiol Res 2005, 54:549-556.

25. Edoff K, Hildebrand C: Neuropeptide effects on rat chondrocytes and perichondrial cells in vitro. Neuropeptides 2003, 37:316-318.

26. Jikko A, Murakami H, Yan W, Nakashima K, Ohya Y, Satakeda H, Noshiro M, Kawamoto T, Nakamura S, Okada Y, Suzuki F, Kato Y: Effects of cyclic adenosine $3^{\prime}, 5^{\prime}$-monophosphate on chondrocyte terminal differentiation and cartilage-matrix calcification. Endocrinology 1996, 137:122-128.

27. Maestroni GJ: Neurohormones and catecholamines as functional components of the bone marrow microenvironment. Ann N Y Acad Sci 2000, 917:29-37.

28. Pongratz G, Straub RH: Role of peripheral nerve fibres in acute and chronic inflammation in arthritis. Nat Rev Rheumatol 2013, 9:117-126.

29. Goldring MB, Goldring SR: Osteoarthritis. J Cell Physiol 2007, 213:626-634.

30. Suri S, Gill SE, Massena dC, Wilson D, McWilliams DF, Walsh DA: Neurovascular invasion at the osteochondral junction and in osteophytes in osteoarthritis. Ann Rheum Dis 2007, 66:1423-1428.

31. Wojtys EM, Beaman DN, Glover RA, Janda D: Innervation of the human knee joint by substance-P fibers. Arthroscopy 1990, 6:254-263.

32. Neame RL, Carr AJ, Muir K, Doherty M: UK community prevalence of knee chondrocalcinosis: evidence that correlation with osteoarthritis is through a shared association with osteophyte. Ann Rheum Dis 2003, 62:513-518.

33. Schaible HG: Mechanisms of chronic pain in osteoarthritis. Curr Rheumatol Rep 2012, 14:549-556.

34. Lehner B, Koeck FX, Capellino S, Schubert TE, Hofbauer R, Straub RH: Preponderance of sensory versus sympathetic nerve fibers and increased cellularity in the infrapatellar fat pad in anterior knee pain patients after primary arthroplasty. J Orthop Res 2008, 26:342-350.

35. Miller LE, Justen HP, Scholmerich J, Straub RH: The loss of sympathetic nerve fibers in the synovial tissue of patients with rheumatoid arthritis is accompanied by increased norepinephrine release from synovial macrophages. FASEB J 2000, 14:2097-2107.

36. Eitner A, Pester J, Nietzsche S, Hofmann GO, Schaible HG: The innervation of synovium of human osteoarthritic joints in comparison with normal rat and sheep synovium. Osteoarthritis Cartilage 2013, 21:1383-1391. 
37. Buma $P$, Verschuren $C$, Versleyen $D$, der KP V, Oestreicher AB: Calcitonin gene-related peptide, substance $P$ and GAP-43/B-50 immunoreactivity in the normal and arthrotic knee joint of the mouse. Histochemistry 1992 98:327-339.

38. Gomez C, Burt-Pichat B, Mallein-Gerin F, Merle B, Delmas PD, Skerry TM, Vico L, Malaval L, Chenu C: Expression of semaphorin-3A and its receptors in endochondral ossification: potential role in skeletal development and innervation. Dev Dyn 2005, 234:393-403.

39. Tolofari SK, Richardson SM, Freemont AJ, Hoyland JA: Expression of semaphorin $3 \mathrm{~A}$ and its receptors in the human intervertebral disc: potential role in regulating neural ingrowth in the degenerate intervertebral disc. Arthritis Res Ther 2010, 12:R1

40. Okubo M, Kimura T, Fujita Y, Mochizuki S, Niki Y, Enomoto H, Suda Y, Toyama $Y$, Okada $Y$ : Semaphorin $3 A$ is expressed in human osteoarthritic cartilage and antagonizes vascular endothelial growth factor 165promoted chondrocyte migration: an implication for chondrocyte cloning. Arthritis Rheum 2011, 63:3000-3009.

41. Opolka A, Straub RH, Pasoldt A, Grifka J, Grässel S: Substance P and norepinephrine modulate murine chondrocyte proliferation and apoptosis. Arthritis Rheum 2012, 64:729-739.

42. Millward-Sadler SJ, Mackenzie A, Wright MO, Lee HS, Elliot K, Gerrard L, Fiskerstrand CE, Salter DM, Quinn JP: Tachykinin expression in cartilage and function in human articular chondrocyte mechanotransduction. Arthritis Rheum 2003, 48:146-156.

43. Karahan S, Kincaid SA, Baird AN, Kammermann JR: Distribution of beta-endorphin and substance $P$ in the shoulder joint of the dog before and after a low impact exercise programme. Anat Histol Embryol 2002, 31:72-77.

44. Millward-Sadler SJ, Wright MO, Davies LW, Nuki G, Salter DM: Mechanotransduction via integrins and interleukin-4 results in altered aggrecan and matrix metalloproteinase 3 gene expression in normal, but not osteoarthritic, human articular chondrocytes. Arthritis Rheum 2000, 43:2091-2099.

45. Howard MR, Millward-Sadler SJ, Vasilliou AS, Salter DM, Quinn JP. Mechanical stimulation induces preprotachykinin gene expression in osteoarthritic chondrocytes which is correlated with modulation of the transcription factor neuron restrictive silence factor. Neuropeptides 2008, 42:681-686.

46. Inoue H, Shimoyama Y, Hirabayashi K, Kajigaya H, Yamamoto S, Oda H, Koshihara $Y$ : Production of neuropeptide substance $P$ by synovial fibroblasts from patients with rheumatoid arthritis and osteoarthritis. Neurosci Lett 2001, 303:149-152.

47. Im HJ, Li X, Muddasani P, Kim GH, Davis F, Rangan J, Forsyth CB, Ellman M, Thonar EJ: Basic fibroblast growth factor accelerates matrix degradation via a neuro-endocrine pathway in human adult articular chondrocytes. J Cell Physiol 2008, 215:452-463

48. Lotz M, Carson DA, Vaughan JH: Substance $P$ activation of rheumatoid synoviocytes: neural pathway in pathogenesis of arthritis. Science 1987, 235:893-895.

49. Tanabe T, Otani H, Mishima K, Ogawa R, Inagaki C: Mechanisms of oxyradical production in substance $P$ stimulated rheumatoid synovial cells. Rheumatol Int 1996, 16:159-167.

50. Lotz M, Vaughan JH, Carson DA: Effect of neuropeptides on production of inflammatory cytokines by human monocytes. Science 1988, 241:1218-1221.

51. Elefteriou F: Neuronal signaling and the regulation of bone remodeling. Cell Mol Life Sci 2005, 62:2339-2349.

52. Schinke T, Liese $S$, Priemel M, Haberland M, Schilling AF, Catala-Lehnen $P$, Blicharski D, Rueger JM, Gagel RF, Emeson RB, Amling M: Decreased bone formation and osteopenia in mice lacking alpha-calcitonin gene-related peptide. J Bone Miner Res 2004, 19:2049-2056.

53. Jiang W, Gao SG, Chen XG, Xu XC, Xu M, Luo W, Tu M, Zhang FJ, Zeng C, Lei GH: Expression of synovial fluid and articular cartilage VIP in human osteoarthritic knee: a new indicator of disease severity? Clin Biochem 2012, 45:1607-1612

54. Niissalo S, Hukkanen M, Imai S, Tornwall J, Konttinen YT: Neuropeptides in experimental and degenerative arthritis. Ann N Y Acad Sci 2002, 966:384-399.

55. Delgado M, Abad C, Martinez C, Leceta J, Gomariz RP: Vasoactive intestinal peptide prevents experimental arthritis by downregulating both autoimmune and inflammatory components of the disease. Nat Med 2001, 7:563-568.
56. Lai LP, Mitchell J: Beta2-adrenergic receptors expressed on murine chondrocytes stimulate cellular growth and inhibit the expression of Indian hedgehog and collagen type X. J Cell Biochem 2008, 104:545-553.

57. Mitchell J, Lai LP, Peralta F, Xu Y, Sugamori K: beta2-adrenergic receptors inhibit the expression of collagen type II in growth plate chondrocytes by stimulating the AP-1 factor Jun-B. Am J Physiol Endocrinol Metab 2011 300:E633-E639.

58. Takarada T, Hojo H, lemata M, Sahara K, Kodama A, Nakamura N, Hinoi E, Yoneda $Y$ : Interference by adrenaline with chondrogenic differentiation through suppression of gene transactivation mediated by Sox 9 family members. Bone 2009, 45:568-578.

59. Jenei-Lanzl Z, Grässel S, Pongratz G, Kees F, Miosge N, Angele P, Straub RH: Norepinephrine inhibits mesenchymal stem cell and chondrogenic progenitor cell chondrogenesis and accelerates chondrogenic hypertrophy. Arthritis Rheum 2014, 66:2472-2481.

60. Li H, Fong C, Chen Y, Cai G, Yang M: Beta-adrenergic signals regulate adipogenesis of mouse mesenchymal stem cells via CAMP/PKA pathway. Mol Cell Endocrinol 2010, 323:201-207.

61. Bjurholm A, Kreicbergs A, Brodin E, Schultzberg M: Substance P- and CGRPimmunoreactive nerves in bone. Peptides 1988, 9:165-171.

62. Hukkanen M, Konttinen YT, Santavirta S, Paavolainen P, Gu XH, Terenghi G, Polak JM: Rapid proliferation of calcitonin gene-related peptideimmunoreactive nerves during healing of rat tibial fracture suggests neural involvement in bone growth and remodelling. Neuroscience 1993, 54:969-979.

63. Lerner UH: Neuropeptidergic regulation of bone resorption and bone formation. J Musculoskelet Neuronal Interact 2002, 2:440-447.

64. Mach DB, Rogers SD, Sabino MC, Luger NM, Schwei MJ, Pomonis JD, Keyser CP, Clohisy DR, Adams DJ, O'Leary P, Mantyh PW: Origins of skeletal pain: sensory and sympathetic innervation of the mouse femur. Neuroscience 2002, 113:155-166.

65. Aro H: Effect of nerve injury on fracture healing. Callus formation studied in the rat. Acta Orthop Scand 1985, 56:233-237.

66. Nordsletten L, Madsen JE, Almaas R, Rootwelt T, Halse J, Konttinen YT, Hukkanen M, Santavirta S: The neuronal regulation of fracture healing. Effects of sciatic nerve resection in rat tibia. Acta Orthop Scand 1994 65:299-304

67. Niedermair T, Kuhn V, Doraneh Gard F, Stange R, Wieskötter B, Beckmann J, Salmen P, Springorum HR, Straub RH, Zimmer A, Grifka J, Grässel S: Absence of substance $P$ and the sympathetic nervous system impact on bone structure and chondrocyte differentiation in an adult model of endochondral ossification. Matrix Biol 2014, 38:22-35.

68. Li J, Kreicbergs A, Bergstrom J, Stark A, Ahmed M: Site-specific CGRP innervation coincides with bone formation during fracture healing and modeling: a study in rat angulated tibia. J Orthop Res 2007, 25:1204-1212.

69. Elefteriou F, Ahn JD, Takeda S, Starbuck M, Yang X, Liu X, Kondo H, Richards WG, Bannon TW, Noda M, Clement K, Vaisse C, Karsenty G: Leptin regulation of bone resorption by the sympathetic nervous system and CART. Nature 2005, 434:514-520.

70. Mukohyama H, Ransjo M, Taniguchi H, Ohyama T, Lerner UH: The inhibitory effects of vasoactive intestinal peptide and pituitary adenylate cyclaseactivating polypeptide on osteoclast formation are associated with upregulation of osteoprotegerin and downregulation of RANKL and RANK. Biochem Biophys Res Commun 2000, 271:158-163.

71. da Silva JA P, Carmo-Fonseca M: Peptide containing nerves in human synovium: immunohistochemical evidence for decreased innervation in rheumatoid arthritis. J Rheumatol 1990, 17:1592-1599.

72. Asmus SE, Parsons S, Landis SC: Developmental changes in the transmitter properties of sympathetic neurons that innervate the periosteum. J Neurosci 2000, 20:1495-1504.

73. Ernsberger $U$ : Role of neurotrophin signalling in the differentiation of neurons from dorsal root ganglia and sympathetic ganglia. Cell Tissue Res 2009, 336:349-384.

74. Mano T, Nishimura N, Iwase S: Sympathetic neural influence on bone metabolism in microgravity (Review). Acta Physiologica Hungarica 2010, 97:354-361.

75. Sherman BE, Chole RA: A mechanism for sympathectomy-induced bone resorption in the middle ear. Otolaryngol Head Neck Surg 1995, 113:569-581.

76. Nixon AJ, Cummings JF: Substance P immunohistochemical study of the sensory innervation of normal subchondral bone in the equine metacarpophalangeal joint. Am J Vet Res 1994, 55:28-33. 
77. Imai S, Tokunaga Y, Maeda T, Kikkawa M, Hukuda S: Calcitonin generelated peptide, substance $P$, and tyrosine hydroxylase-immunoreactive innervation of rat bone marrows: an immunohistochemical and ultrastructural investigation on possible efferent and afferent mechanisms. J Orthop Res 1997, 15:133-140.

78. Ogino S, Sasho T, Nakagawa K, Suzuki M, Yamaguchi S, Higashi M, Takahash K, Moriya $H$ : Detection of pain-related molecules in the subchondral bone of osteoarthritic knees. Clin Rheumatol 2009, 28:1395-1402.

79. Fortier LA, Nixon AJ: Distributional changes in substance P nociceptive fiber patterns in naturally osteoarthritic articulations. J Rheumatol 1997, 24:524-530.

80. Liu D, Jiang LS, Dai LY: Substance $P$ and its receptors in bone metabolism. Neuropeptides 2007, 41:271-283.

81. Lerner UH: Deletions of genes encoding calcitonin/alpha-CGRP, amylin and calcitonin receptor have given new and unexpected insights into the function of calcitonin receptors and calcitonin receptor-like receptors in bone. J Musculoskelet Neuronal Interact 2006, 6:87-95.

82. Aitken SJ, Landao-Bassonga E, Ralston SH, Idris Al: Beta2-adrenoreceptor ligands regulate osteoclast differentiation in vitro by direct and indirect mechanisms. Arch Biochem Biophys 2009, 482:96-103.

83. Ma Y, Krueger JJ, Redmon SN, Uppuganti S, Nyman JS, Hahn MK, Elefteriou F: Extracellular norepinephrine clearance by the norepinephrine transporter is required for skeletal homeostasis. J Biol Chem 2013, 288:30105-30113.

84. Cornish J, Callon KE, Bava U, Kamona SA, Cooper GJ, Reid IR: Effects of calcitonin, amylin, and calcitonin gene-related peptide on osteoclast development. Bone 2001, 29:162-168.

85. Li J, Wang Y, Li Y, Sun J, Zhao G: The effect of combined regulation of the expression of peroxisome proliferator-activated receptor-gamma and calcitonin gene-related peptide on alcohol-induced adipogenic differentiation of bone marrow mesenchymal stem cells. Mol Cell Biochem 2014, 392:39-48.

86. Goto T, Nakao K, Gunjigake KK, Kido MA, Kobayashi S, Tanaka T: Substance $P$ stimulates late-stage rat osteoblastic bone formation through neurokinin-1 receptors. Neuropeptides 2007, 41:25-31.

87. Wang L, Zhao R, Shi X, Wei T, Halloran BP, Clark DJ, Jacobs CR, Kingery WS: Substance $P$ stimulates bone marrow stromal cell osteogenic activity, osteoclast differentiation, and resorption activity in vitro. Bone 2009, 45:309-320.

88. Adamus MA, Dabrowski ZJ: Effect of the neuropeptide substance $\mathbf{P}$ on the rat bone marrow-derived osteogenic cells in vitro. J Cell Biochem 2001, 81:499-506.

89. Kingery WS, Offley SC, Guo TZ, Davies MF, Clark JD, Jacobs CR: A substance $P$ receptor (NK1) antagonist enhances the widespread osteoporotic effects of sciatic nerve section. Bone 2003, 33:927-936.

90. Ding WG, Zhang ZM, Zhang YH, Jiang SD, Jiang LS, Dai LY: Changes of substance $P$ during fracture healing in ovariectomized mice. Regul Pept 2010, 159:28-34.

91. Yoo YM, Kwag JH, Kim KH, Kim CH: Effects of neuropeptides and mechanical loading on bone cell resorption in vitro. Int J Mol Sci 2014, 15:5874-5883.

92. Persson E, Lerner UH: The neuropeptide VIP potentiates IL-6 production induced by proinflammatory osteotropic cytokines in calvarial osteoblasts and the osteoblastic cell line MC3T3-E1. Biochem Biophys Res Commun 2005, 335:705-711.

93. Kondo H, Guo J, Bringhurst FR: Cyclic adenosine monophosphate/protein kinase A mediates parathyroid hormone/parathyroid hormone-related protein receptor regulation of osteoclastogenesis and expression of RANKL and osteoprotegerin mRNAs by marrow stromal cells. J Bone Miner Res 2002, 17:1667-1679.

94. Persson $\mathrm{E}$, Lerner UH: The neuropeptide VIP regulates the expression of osteoclastogenic factors in osteoblasts. J Cell Biochem 2011, 112:3732-3741.

95. Yu XJ, Ren XH, Xu YH, Chen LM, Zhou CL, Li CY: Vasoactive intestinal peptide induces vascular endothelial growth factor production in human HaCaT keratinocytes via MAPK pathway. Neuropeptides 2010, 44:407-411.

96. Mei G, Zou Z, Fu S, Xia L, Zhou J, Zhang Y, Tuo Y, Wang Z, Jin D: Substance $P$ Activates the Wnt signal transduction pathway and enhances the differentiation of mouse preosteoblastic MC3T3-E1 cells. Int J Mo/ Sci 2014, 15:6224-6240
97. Mei G, Xia L, Zhou J, Zhang Y, Tuo Y, Fu S, Zou Z, Wang Z, Jin D Neuropeptide SP activates the WNT signal transduction pathway and enhances the proliferation of bone marrow stromal stem cells. Cell Biol Int 2013, 37:1225-1232.

98. Mrak E, Guidobono F, Moro G, Fraschini G, Rubinacci A, Villa I: Calcitonin gene-related peptide (CGRP) inhibits apoptosis in human osteoblasts by beta-catenin stabilization. J Cell Physiol 2010, 225:701-708.

doi:10.1186/s13075-014-0485-1

Cite this article as: Grässel: The role of peripheral nerve fibers and their neurotransmitters in cartilage and bone physiology and pathophysiology. Arthritis Research \& Therapy 2014 6:485. 